\title{
Fast versus slow bandaid removal: a randomised trial
}

\author{
Jeremy S Furyk, Carl J O'Kane, Peter J Aitken, Colin J Banks and David A Kault
}

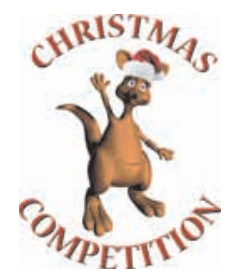

A pplying dressings to wounds is a common practice throughout the world, both in and out of hospitals. At times, removing such dressings can be more painful than the wound itself. ${ }^{1}$ Research on dressing removal has often focused on expensive new products ${ }^{2}$ and, although speed of dressing removal has been controlled for in some studies ${ }^{2}$ (implying that speed is a factor), we are not aware of any research directed specifically at different speeds of dressing removal. There are Internet sites addressing how to reduce the pain associated with removal of certain brands of dressings, such as Band-Aid (Johnson \& Johnson, New Brunswick, NJ, USA) (eg, http://www.wikihow.com/ Remove-a-Band-Aid); however, there is no consensus on the issue of speed.

The pain of dressing removal is thought to be related to the mechanical stripping of the stratum corneum from the underlying epidermal and dermal cells. ${ }^{3}$ However, the perception of pain is complex - it is a multifactorial experience influenced by culture, previous pain events, beliefs, mood and ability to cope. ${ }^{4}$

Simple dressings are known by many different names, such as sticking plasters. Band-Aid is one of the most popular and best-selling brands of simple dressings worldwide; it is used so frequently both in domestic and health care settings that the brand name has been adopted for generic usage.

The two most common methods of bandaid removal are slow bandaid removal (SBAR) and fast bandaid removal (FBAR). Proponents of the slow technique advocate that minimising the noxious stimulus is preferable, despite exposing the individual to longer total time of stimulus. With the fast technique, shorter exposure to the noxious stimulus may be perceived as less painful, despite short intense stimulation of pain-perceiving nociceptors.

We aimed to compare these two common methods of bandaid removal and to definitively answer the question: which method of removal, fast or slow, causes less pain?

\section{METHODS}

The study was a prospective, randomised, crossover trial comparing FBAR with SBAR in healthy volunteers. It was conducted on 4 August 2009 at James Cook

University, Townsville,

Queensland.

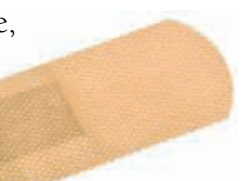

\section{ABSTRACT}

Objective: To determine whether slow or fast bandaid removal is less painful.

Design, setting and participants: A prospective, randomised, crossover trial was carried out at James Cook University, Townsville. Participants were healthy volunteers from Years 2 and 3 of the James Cook University medical school program.

Interventions: Medium-sized bandaids were applied bilaterally in three standard body locations and removed using slow and fast techniques.

Main outcome measures: Pain scores were assessed using an 11-point verbal numeric pain scale.

Results: 65 participants were included in the study. The overall mean pain score for fast bandaid removal was 0.92 and for slow bandaid removal was 1.58 . This represents a highly significant difference of $0.66(P<0.001)$.

Conclusion: In young healthy volunteers, fast bandaid removal caused less pain than slow bandaid removal.

MJA 2009; 191: 682-683

The study participants were a convenience sample of healthy volunteers from the second and third years of a 6-year, undergraduate medical school program at James Cook University. Inclusion criteria were age greater than 18 years and ability to provide informed consent. Exclusion criteria included documented or suspected allergy to adhesive dressings, and chronic pain or anxiety disorder. Written informed consent was obtained from all participants. The participants were not coerced, although pizza was available for them. Participants were also made aware of the benefits to humanity that the study would potentially provide.

The brand of dressings used was Band-Aid (Johnson \& Johnson); medium-sized dressings were used. These were not supplied by the manufacturer. Dressings were applied bilaterally in three standard body locations: the upper arm over the deltoid, the dorsum of the hand, and immediately superior to the medial malleolus of the ankle. The participants were randomly assigned sequentially numbered data collection sheets in sealed opaque envelopes to determine which removal technique (FBAR or SBAR) would be tested first and which side of the body (left or right) would be tested first. This sequence of bandaid removal was maintained for all three locations in each individual participant. The random sequence was determined by use of standard random number tables. Data on age, sex, the participants' preconceptions about which method would be more painful, ethnicity and amount of body hair (assessed by using a 5-point scale, where 1 represented "no hair" and 5 represented "most hairy") were also collected.

The two operators (CJO and CJB) attended a 1-hour training session on dressing removal before commencement of the study. FBAR consisted of a single rapid movement, whereas SBAR consisted of dressing removal over a 2 -second period. One of us (JSF) observed all dressing removals to ensure consistency of technique.

The primary outcome was pain score assessed using an 11-point, verbal numeric pain scale, where 0 represented "no pain" and 10 represented "worst pain imaginable". Immediately after dressing removal, participants were asked to rate the pain experienced.

Data were entered into an Excel spreadsheet (Microsoft, Redmond, Wash, USA) and analysed using Public Domain Statistics (James Cook University, Townsville, Qld) and SPSS version 14.0 (SPSS, Chicago, Ill, USA). The average differences in pain scores, for SBAR versus FBAR and for each individual participant, were tested for significance. The overall outcome was assessed using a single-sample $t$ test, to test whether the measures for individuals were significantly different from zero (equivalent to a paired-sample $t$ test). Other results were analysed using independent-sample $t$ tests and simple regression.

A minimum sample size of 60 was needed to demonstrate a difference in mean overall pain scores of 0.5 between SBAR and FBAR, assuming a variance in the data to be analysed of 2, statistical power of $80 \%$ (type 2 error of $20 \%$ ) and an alpha value of 0.05 . We thought 
pain scores for dressing removal would be at the lower end of the pain scale and, although the level of clinical significance in this range is not known, we assumed a change of 0.5 to be clinically significant.

The study protocol was approved by the Townsville Health Service District Institutional Ethics Committee and conforms to the provisions of the Declaration of Helsinki. ${ }^{5}$ There was no manufacturer involvement in the study.

\section{RESULTS}

Sixty-five participants were included in the study, of whom 35 were female (54\%) and 48 were of European ancestry (74\%); the mean age of participants was 20.1 years (range 1830 years). Most participants (49/65; 75\%) believed that SBAR would be more painful than FBAR. The mean body hair score for men was 2.1 and the mean for women was 1.3 $(P<0.001)$.

The mean overall pain score for FBAR was 0.92 , and for SBAR was 1.58. This represents a highly significant difference of 0.66 $(P<0.001)$. However, FBAR was not associated with lower pain scores than SBAR for all participants. An analysis of individual predictor variables indicated that low body hair score, preconception that SBAR would be more painful than FBAR, and testing FBAR before SBAR were all significantly related to lower pain scores for FBAR compared with SBAR $(P=0.01, P=0.03$ and $P=0.03$, respectively). Increasing age, operator and ethnicity were less convincingly related $(P=0.11, P=$ 0.08 and $P=0.10$, respectively). There was no appreciable difference due to the left or right side being tested first. Mean overall pain scores for women were significantly lower than for men (0.91 v 1.64, respectively; $P<0.001)$.

\section{DISCUSSION}

Our results show that FBAR was less painful than SBAR. This is consistent with the preconceptions of most of our sample. A high body hair score was, not surprisingly, associated with higher pain scores, and it seemed that preconceptions also had an appreciable effect. Several other aspects of our data may require further investigation. The pain experience is a complex and incompletely understood process that incorporates many aspects of patients' social and cultural beliefs, as well as previous experiences. ${ }^{4}$ Our observation that preconceptions were associated with pain scores should not therefore be surprising.

The association between increasing age and higher pain scores is interesting, although this did not reach statistical significance. Our sam- ple consisted of young healthy adults; therefore our results may not be applicable to other age groups such as children and older people. The operator effect was also interesting. Although FBAR was associated with lower pain scores in participants belonging to both operator groups, the fact that the scores were different between groups suggests that removal of dressings may be operator-dependent, and there may be skilled bandaid removers and less-skilled or unskilled bandaid removers.

The lower mean pain score for women may be due to a higher pain tolerance, although men did have a significantly higher body hair score.

It is also important to note that our participants were healthy volunteers with no wounds, thus pain was associated with minor skin damage only and not associated with wound pain. These results would therefore not be applicable to patients with wounds, particularly chronic wounds and ulcers, which may adhere to Band-Aid brand dressings and other brands of simple dressings.

Our study had other limitations. These included the inability to blind the participants (in terms of which removal technique and which side of the body would be tested first) and preconceived ideas regarding the technique that would be more painful, both of which may have biased our results. However, the use of randomisation and a crossover design should have minimised this inherent bias. In addition, scoring pain is an imprecise science and there is no perfect pain assessment tool. The verbal numeric pain scale is a commonly used pain assessment tool and has been validated for use in emergency settings. ${ }^{6}$ It is possible that it may be less reliable and reproducible at the lower ends of the pain scale.

We used human rather than mechanical operators to conduct both SBAR and FBAR. Although this may have led to human error and bias, we chose this method to increase external validity and ability to generalise our results. Although mechanised dressing removers may be available in the research setting, they are unlikely to become widely available for routine use in the home or hospital. Therefore use of mechanised dressing removers would have limited the conclusions that could be drawn from our study. We would have liked to have recorded video samples of SBAR and FBAR to ensure standardisation of removal speed, but this was not possible owing to insufficient budget.

In a sample of young healthy volunteers, we found FBAR caused less pain than SBAR. A high body hair score and preconception that SBAR would be more painful were also posi- tively associated with pain scores. Our results may not be applicable to different types of dressings or specific wounds, and further research is needed to address these issues. However, we hope that our results will assist parents, carers and health care staff in adopting the least painful method of bandaid removal.

\section{COMPETING INTERESTS}

None identified.

\section{AUTHOR DETAILS}

Jeremy S Furyk, MB BS, FACEM, MPHTM, Emergency Physician, ${ }^{1}$ and Associate ${ }^{2}$

Carl J O'Kane, FACEM, Emergency Physician, ${ }^{1}$ and Associate ${ }^{2}$

Peter J Aitken, MB BS, FACEM, EMDM, Emergency Physician, ${ }^{1}$ Associate, ${ }^{2}$ Associate Professor, ${ }^{3}$ and Noel Stevenson Fellow ${ }^{4}$ Colin J Banks, MB BS, FACEM, Emergency Physician, ${ }^{1}$ and Associate ${ }^{2}$

David A Kault, MB BS, BSc, PhD, Senior Lecturer $^{5}$

1 Department of Emergency Medicine, Townsville Hospital, Townsville, QLD.

2 Townsville Institute of Research in Emergency Departments (TIRED), Townsville, QLD.

3 Anton Breinl Centre, James Cook University, Townsville, QLD.

4 Queensland Emergency Medicine Research

Foundation, Brisbane, QLD.

5 School of Engineering and Physical Sciences, James Cook University, Townsville, QLD.

Correspondence:

jeremy_furyk@health.qld.gov.au

\section{REFERENCES}

1 Woo KY, Harding K, Price P, Sibbald G. Minimising wound-related pain at dressing change: evidence-informed practice. Int Wound J 2008; 5: 144-157.

2 Dykes $\mathrm{P}$, Heggie R. The link between the peel force of adhesive dressings and subjective discomfort in volunteer subjects. J Wound Care 2003; 12: 260-262.

3 Dykes P, Heggie R, Hill S. Effects of adhesive dressings on the stratum corneum of the skin. $J$ Wound Care 2001; 10: 7-10.

4 Australian and New Zealand College of Anaesthetists and Faculty of Pain Medicine. Acute pain management: scientific evidence (second edition). Melbourne: ANZCA, 2005. http:// www.nhmrc.gov.au/_files_nhmrc/file/publications/synopses/cp104.pdf (accessed Aug 2009).

5 World Medical Association Declaration of Helsinki - Ethical Principles for Medical Research Involving Human Subjects. http://www.wma.net/ en/30publications/10policies/b3/index.html (accessed Sep 2008)

6 Holdgate A, Asha S, Craig J, Thompson J. Comparison of a verbal numeric rating scale with the visual analogue scale for the measurement of acute pain. Emerg Med (Fremantle) 2003; 15: 441 446.

(Received 3 Oct 2009, accepted 19 Oct 2009) $\square$ 